\title{
Potential advantages and disadvantages of telemedicine: A literature review from the perspectives of patients, medical personnel, and hospitals
}

\author{
Lie Rebecca Yen Hwei, Gilbert Sterling Octavius* \\ Faculty of Medicine, University of Pelita Harapan, Tangerang, Indonesia
}

SUBMITTED: 22 February 2021

REVISED: 6 May 2021

ACCEPTED: 2 June 2021

KEYWORDS

Hospitals perspective

Medical personnel

Patients perspective

Telemedicine
ABSTRACT With the increase in the aging population around the world, the medical field is also changing in response. The number one cause of mortality in the aging population is non-communicable diseases such as diabetes mellitus, heart disease or kidney failure. Advancements in technology make it possible for diagnoses and treatments to be no longer confined physically but they can be done virtually. However, there are several drawbacks in fully utilizing telemedicine such as hesitancy from the medical personnel and unclear law requirements. In this review, we discuss the advantages and disadvantages of telemedicine from three perspectives of the patients, medical personnel, and hospitals.

(c) The Journal 2021. This article is distributed under a Creative Commons Attribution-ShareAlike 4.0 International license.

\section{Introduction}

The aging population around the world is increasing. It affects individuals and their communities socially, both individuals as elderly people and their country economically by increasing the prevalence of chronic conditions associated with aging, as well as affecting individuals' capability to do daily activities. Chronic health problems have major effects on the quality of life of an individual and can consume a considerable amount of money and time for health and social services. ${ }^{1}$

The cost for treatment is increasing every year. The estimated costs of hospital beds per diem in Indonesia in 2005 were as follows: \$30.36 for primary hospitals, $\$ 39.61$ for secondary hospital, and $\$ 54.10$ for tertiary hospitals. The estimated patient costs in 2005 were as follows: $\$ 9.25$ for primary hospitals, $\$ 13.12$ for secondary hospitals, and $\$ 19.41$ for tertiary hospitals. The costs estimated above do not include drugs and diagnostics. ${ }^{2}$ Mortality number of children age 5 and below showed a significant difference between the very poor and the wealthy with a mean difference of $42.1 \% .^{3}$ The gap in health

*Correspondence: sterlinggilbert613@hotmail.com

Faculty of Medicine, University of Pelita Harapan, MH Thamrin Boulevard 1100, Klp Dua, Tangerang 15811, Indonesia services shows clear disparities in the medical world where costs often determine a person's ability to seek treatment.

This dilemma is exacerbated by the fact that Indonesia still lacks healthcare professionals. In accordance with 2014 data, Indonesia only has 0.46 physicians, 0.70 nurses, 0.54 midwives, 0.02 dentists, and 0.05 pharmacists per 1,000 population. ${ }^{4}$ According to the World Health Organization (WHO) recommendations, the ideal doctor-patient ratio is $1: 600$, making the condition of the health system in Indonesia one of the worst in Southeast Asia. ${ }^{5}$

In an effort to optimize health services in developing countries such as Indonesia, it is necessary to collaborate with other sectors that can support the health system including science and technology. Telemedicine can be used to meet these basic healthcare needs. It can provide healthcare access to those in Indonesia's remote areas while also allowing healthcare practitioners to provide quality care for their patients through cross-city consultations.

The use of telemedicine as a means to treat the patient virtually dates back to the late 1950s and early 1960s, when a closed-circuit television connection was developed between the Nebraska Psychiatric Institute and Norfolk State Hospital for 
psychiatric consultations. From this time, the use of telemedicine has broadened into many types of services and has been used in different departments, from radiology to intensive care units (ICU). ${ }^{6}$ As a way to increase quality and safety in patient care delivery, information can be collected, processed, retrieved and exchanged by various disciplines. ${ }^{7}$

Telemedicine, also known as Telehealth, is the use of medical information, which is exchanged from one place to another by electronic communication with the purpose of increasing a patient's health. ${ }^{8}$ Based on the types, telemedicine can be categorized into two, which are the synchronous and asynchronous types. Synchronous telemedicine or real-time telehealth is a telemedicine service that involves audiovisual technology that enables individuals to communicate in real time through a video conference site. This method requires the user to have at least a video camera, sound system, computer screen, and safe, high speed Internet connection to transmit data between locations. Because of these needs, this type of telemedicine relies on the availability of a good video conference facility, which is usually available only on the institutional level.

Asynchronous telemedicine or store-andforward telemedicine is the process of data transmission without the need of synchronized interactions between individuals. This type of telemedicine involves digital data collections such as electrocardiogram (ECG), spirometry results, and radiology pictures in one location, which are sent to another location for assessment by professional healthcare workers. Technology required by asynchronous telemedicine includes the equipment, with which the digital data can be captured, stored, and downloaded to be transmitted to remote areas. Digital files can be videos from digital camera, voice notes, or electronic medical records. Asynchronous telemedicine offers lower costs in building infrastructures than synchronous telemedicine and provides more time for healthcare professional to give their input on the data. ${ }^{9}$

\section{Method}

The authors have chosen the review duration over a period of 25 years, starting from the year 1996 to
2021 as the time horizon. The reason for selecting this time horizon is to gather the articles that are still relevant until the time of writing. Various journals and conferences have a plethora of literature relating to the advantages and disadvantages of telemedicine. The authors searched for the articles related to the advantages and disadvantages of telemedicine in several publication portals, such as Google Scholar, PubMed Central, Science Direct, and Springer. For the search criteria, the authors

\section{Result}

\subsection{Advantages}

\subsubsection{Patients}

Telemedicine can be used as a way of providing information for both the patients and the general public. Through telemedicine, the patients will be able to understand the nature of their disease, prognosis associated with the disease, the effect that they might experience in undergoing treatment, and the reasons why the healthcare professionals ask them to do certain examinations. Telemedicine could provide the basis for mutual decision-making between patients and healthcare professionals, encouraging patients and promoting self-help once patients have gained such understanding. Therefore, it is critical that the information is accurate and case-oriented. Telemedicine can also be used to provide information in a form of health promotion or health education to people, schools, and healthcare centers. ${ }^{10}$

A study by Sorwar et al. found that the time a patient spent to access healthcare was reduced by $56 \%$ when using telemedicine compared to conventional health services. They also stated that the average maintenance cost was cut by $94 \% .{ }^{11}$ One systematic review of 22 randomized controlled trials conducted by Andrew et al. showed that despite the decreased number of face-to-face consultations, the patients' satisfaction and adherence to therapy remains high (86\%). ${ }^{12}$ The benefits of telehealth were recognized by individuals engaging in telehealth in Saudi Arabia as enhancing the standard of treatment, improving access to healthcare, and saving both time and money. ${ }^{13}$ 
Another study by Akiyama and Yoo showed that the frequency of doctor visits decreased to once a week compared to before (twice a week). Cost of palliative care also decreased, saving $\$ 5,000$ per person per year compared to conventional health care services. ${ }^{11}$ Dick et al. found that in pediatric consultations, the patients experienced a high-level of comfort in using telemedicine consultations and in the same time period, saving costs of $\$ 1,000$ per person. ${ }^{14}$ Shore et al. reported that the use of telehealth could lead to increased efficiency and reduction of research cost among rural, remote, and underserved populations. ${ }^{15}$

The level of use of a web-based telemedicine system was found to be associated with baseline patient awareness of health, self-efficacy of treatment, and income. The telemedicine system is available widely, but the use of it depends on the patients' health knowledge related to cardiovascular risk. When they feel more in charge of their conditions, they are more likely to take further measures to support their own well-being. Educating patients on health problems motivates them to track their progress and engage more with their physicians, thereby improving their cardiovascular risk. ${ }^{16} \mathrm{~A}$ systematic review conducted by Polisena et al. showed that home telemonitoring can provide congestive heart failure (CHF) patients with improved clinical results compared to conventional treatment. Home telemonitoring was also found to decrease hospitalization rates, the number of emergency visits to the emergency room, reduce all-cause of CHFrelated events, and lower mortality rates. ${ }^{17}$

Statistically, the centralized high-intensity active tele-ICU model has shown dramatically improved clinical outcomes of the patients. There is a high degree of variability in how tele-ICU coverage is provided, which can influence patients' outcomes and safety. A few of the variables are the location (urban or rural) and open or closed unit physician coverage. The tele-ICU offers a way for these patients to provide conventional bedside care while increasing the care and quality of care. As part of the critical care delivery system, the tele-ICU program is able to address insufficient access to critical care services, reduce the level of inconsistency among healthcare providers, improve patient's results, and decrease costs. As the technology becomes better and more accessible, the patient's outcomes and safety also improve. $^{18}$

In less developed, more remote areas where there are even fewer specialists, teleophthalmology often increases equalities in the health services available. This results in a higher number of patients being screened and diagnosed. Incorporating ophthalmology examination in a smartphone application is also available. It can measure visual acuity, assisting in diagnosing and treating several eye conditions such as glaucoma or amblyopia. This approach helps the patients to receive appropriate treatment and referral to specialists when needed. ${ }^{19}$

\subsubsection{Health Professionals}

Whited et al. conducted a study by giving out surveys that were part of a randomized controlled trial on the use of teledermatology. The referring clinicians found that the referred patients received timely consultation if referred using teledermatology compared to the traditional consultation. The dermatologists agreed that teledermatology services spent less time compared to the face-toface consultations. The consultant dermatologists were also satisfied with the use of teledermatology and unitedly agreed that teledermatology made the triage process easier to be done. ${ }^{20} \mathrm{~A}$ study conducted by Barrufadi et al. on the use of teleconsultations in orthopedic departments showed that the physicians saw this technology as an opportunity to improve one's knowledge and overall, while both the orthopedic specialists and remote physicians were satisfied with the system. ${ }^{21}$

Teleophthalmology gives access not only to the patients and healthcare providers but also students and other doctors. It promotes accessibility and the sharing of expertise between young doctors in rural areas and the specialists in hospitals and universities. The use of the technology also enables better communication between the healthcare providers and the patients, hence, increasing patients' compliance. $^{19}$

Telecytopathology enables the pathologists to assess the fine needle aspiration (FNA) remotely and to avoid commuting to multiple locations for the 
procedures. Through experiments in the Acibadem Health Group, it was found that various forms of cytology can be examined at no unnecessary expenses, creating national and international levels of consultation between specialists with results that can be compared with routine cytology in other centers. ${ }^{22}$

The use of automated short message service (SMS) and applications-based interventions in surgical settings have also brought positive results. Patients often have difficulties in remembering the details of post-surgical instructions due to one reason or another. By using the applications, automated information delivery can be helpful to provide reminders to the patients at his or her own comfort at home. This approach increased patients' adherence to the instructions, improving the clinical outcomes. Telemedicine also helps the healthcare providers be better informed about the patient's current condition and monitor the patient's symptoms, if any are present. The symptom monitoring through application interventions decreases the need for hospital visits, creates successful rapid triage and treatment warnings, making the tasks easier for healthcare professionals. ${ }^{23}$

Healthcare professionals were satisfied with the use of telemedicine in specialized palliative care because they were able to interact with each other and keep the conversation open both ways between the patients and themselves. Video consultations also helped the healthcare professionals to communicate routinely with the patients and their families, and to discuss further treatment based on their meeting. The healthcare professionals were able to assess the patient's physical or mental changes over time easily through the video consultation. ${ }^{24}$

\subsubsection{Hospitals}

The systematic review conducted by Lu et al. showed that the use of SMS and applicationsbased interventions have a potential of significant cost savings. For example, the emergency room visits decreased. The patients did not have to come back to the hospital because their problems were solved by the applications-based interventions. simpler symptoms in low-risk patients were also resolved without clinic visits; thereby, saving costs and resources. On the other hand, the monitoring of high-risk patients will also save costs since it can enable a method of early detection of possible complications. ${ }^{23}$

A study conducted by Maarop, Singh and Win found that implementation of teleconsultation in Malaysia brought many benefits to healthcare providers, organizations, and patients. The benefits were then categorized into supportive and critical benefits. Supportive benefits are the use of technology to support consultation and referral efficiently while critical benefits are the use of technology to support hospital organizations and health care facilities to provide health care services for underserved and critical patients. Using the teleconsultation approach, the hospital and other health care facilities were able to give advice for critical patients without waiting for one specific specialist to respond to the call. They had better utilization of their resources, reduced mortality and morbidity, and reduced patient movement. All in all, the organizations are able to save costs and resources more than before. ${ }^{25}$

\subsection{Disadvantages}

\subsubsection{Patients}

Although the telemedicine usage proved to have advantages, it also has disadvantages. The decreasing quality of patient-doctor relationship cannot be ignored. The patients who have some disability such as decreased hearing or seeing ability might experience difficulty in using telemedicine. Old people who grew up seeing the monitor as an experience of watching television tend to not feel like the doctor actually saw and listened to them. This can be a barrier for the patient to receive consultation properly. Teleconsultation also does not allow the doctor to perform the physical examination so that it might feel like that the doctor is not performing a thorough consultation. ${ }^{10}$

Difficulties in logistic aspects of teleophthalmology, together with technological errors and poor communication can contribute to less efficient services. Accuracy of diagnosis and then potential treatment for the patients can also 
be affected. There are also privacy issues regarding the accumulated data. ${ }^{19}$ Using SMS or applications as a reminder can also cause usage fatigue, where the patients may stop responding to the interface or worse, stop using it at all. ${ }^{23}$

Compared to face-to-face consultations, telemedicine has increased risks related to privacy and security. Even though there are standards and regulations that protect telehealth platforms, no platform is $100 \%$ safe from breaches. Both the data provider (patients) and the service provider (healthcare professionals) need to agree on some terms and conditions related to the privacy and security aspects of telemedicine. Telehealth providers must take responsibility for maintaining compliance with legislation, patient confidentiality, and system security throughout all times. The accuracy of data transmission can also affect the diagnosis and treatment provided by the healthcare professionals. One report studying the accuracy of physical function measurements showed that the validity and reliability of fine motor task measurements are influenced by the Internet bandwidth. ${ }^{26}$

\subsubsection{Health Professionals}

Telemedicine might be interpreted as a threat to their current job or autonomy in the remote places when the healthcare professionals in remote areas consult a case to the specialist in bigger cities, or worse they will be perceived as mere technicians. There are also problems concerning the quality of available medical information on the Internet. The information can be textbook-style information that was produced by medical schools and institutions, abstracts or articles in biomedical journals, and health pamphlets and articles intended for general public that were produced by individuals and organizations. The last of the three can be concerning as the content might be biased, inaccurate, or even, misleading. ${ }^{10}$

Symptom monitoring by mobile-health or $m$-health interventions can be a burden to the medical team because some of the patients might give out false alarms that require immediate attention to their non-emergency situations. ${ }^{23}$

Telehealth work poses numerous concerns about malpractice liability, including informed consent, guidelines and procedures for practices that are the appropriate standard of care, supervision criteria for non-physician care providers, and the availability of insurance coverage for professional liability. As the use of telehealth grows, it is also prone to fraud and abuse so that strict laws will be needed to keep the practices legitimate and correct. Prescribing various controlled substances for certain conditions also needs to be addressed. While the physician can directly write the prescription in faceto-face consultations, they cannot do the same in teleconsultation. There are controlled substances that cannot be prescribed, distributed, and delivered without a valid prescription which need at least one face-to-face consultation. Therefore, the distribution of certain medications is restricted. ${ }^{26}$

\subsubsection{Hospitals}

Implementation of telemedicine can bring issues to the hospitals and other health care facilities related to infrastructure planning and development, certain telecommunication regulations, reimbursement systems with the government, licensure and credentialing, medical malpractice liability, and confidentiality. There might also be difficulties in persuading the healthcare workers to change the way they work currently to adapt to the telemedicine system. ${ }^{10}$

El-Mahalli et al. showed that budget and infrastructure are the main barriers in implementing telemedicine in the hospitals in Saudi Arabia. Lack of knowledge on telemedicine and its benefits, and lack of time to learn how to adopt the technology contribute to more training and workshops to be done for the adopters, resulting in increasing costs for the health care facilities. ${ }^{13}$

In tele-ICU programs, malpractice by remote healthcare professionals has become an increasing concern. There are many aspects to be considered outside the requirement for licensure, credentialing, privileging, and malpractice liability, such as issues of jurisdiction and clinical practice regulations. ${ }^{18}$

Lack of reimbursement has also become a problem in adopting telemedicine since the reimbursement might cover only some of the aspects of telemedicine while in practice, many have to be 
done. ${ }^{26}$

\subsection{Telemedicine During the COVID-19 pandemic}

The outbreak of the Coronavirus Disease - 2019 (COVID-19) has become an international concern. The viral infection causes respiratory infections ranging from common colds to more serious illnesses. ${ }^{27}$ The initial symptoms of COVID-19 infection could be dry cough, fever, or difficulty in breathing. ${ }^{28}$ Elderly people and those with underlying comorbidities such as hypertension, diabetes, and heart disease are more likely to have severe signs and symptoms. ${ }^{27}$ Many countries have implemented social-distancing rules to reduce person-to-person interactions since limiting social contact is an essential factor in slowing down the spread of the virus. ${ }^{29}$ To meet both the critical needs of COVID-19 patients and other individuals who need healthcare services, more creative solutions are needed. ${ }^{30}$ Technological developments in the form of telemedicine introduce alternative options in this regard.

Although the ultimate approach to mitigating COVID-19's spread would be multifaceted, telemedicine is one of the effective ways to use current technologies to enable efficient delivery of services while minimizing the risk of direct exposure from person to person. The use of telemedicine in the sense of epidemic conditions has the ability to improve epidemiological interventions, disease prevention, and clinical case management studies. ${ }^{29,31,32}$

\section{Conclusion}

Despite its advantages, telemedicine applications and approaches still have several flaws which deter their use clinically. Therefore, it is imperative for all sectors to work together to implement, execute, and develop telemedicine to better serve the patients' needs. Governments need to tackle the legal aspects of telemedicine while hospitals and medical personnel need to work hand in hand to maximize the clinical use of telemedicine.

\section{References}

1. Wade R, Shaw K, Cartwright C. Factors affecting provision of successful monitoring in home telehealth. Gerontology. 2012;58:371-7.

2. Adam T, Evans DB, Murray CJL. Econometric estimation of country-specific hospital costs. Cost Eff Resour Alloc. 2003;1:3.

3. World Health Organization. State of Health Inequality: Indonesia [Internet]. Switzerland; 2017. Available from: https://www.who. int/docs/default-source/gho-documents/ health-equity/12-dec-final-final-17220-stateof-health-inequality-in-indonesia-for-web. pdf?sfvrsn=54ae73ea_2

4. Mahendradhata $Y$, Trisnantoro $L$, Listyadewi $S$, Soewondo P, Marthias T, Harimurti P, et al. The Republic of Indonesia Health System Review, Health Systems in Transition. WHO Regional Office for South-East Asia. 2017;7:120.

5. Rakhmat MZ, Tarahita D. Floating hospitals: Meeting healthcare gaps in Indonesia [Internet]. 2017. Available from: https://www.asiasentinel. $\mathrm{com} / \mathrm{p} /$ floating-hospitals-healthcare-gapsindonesia

6. IOM (Institute of Medicine). The Role of Telehealth in an Evolving Health Care Environment: Workshop Summary. Washington, D.C.: The National Academies Press; 2012.

7. O'Connell P. Advantages and challenges to using telehealth medicine. Glob J Med Res. 2015;15:5.

8. Tuckson R V, Edmunds M, Ph D, Hodgkins ML. Telehealth. 2017;1585-92.

9. Deshpande A, Khoja S, McKibbon A, Jadad A. Overview of assessments of real-time (synchronous) and asynchronous telehealth [Technology overview number 38]. Can Agency Drugs Technol Heal. 2008;1-58.

10. Hjelm NM. Benefits and drawbacks of telemedicine. J Telemed Telecare. 2005;11:6070.

11. Atmojo JT, Sudaryanto WT, Widiyanto A, Ernawati E, Arradini D. Telemedicine, cost effectiveness, and patients satisfaction: A systematic review. J Heal Policy Manag. 2020;5:103-7.

12. Andrews G, Cuijpers $P$, Craske MG, McEvoy $P$, Titov N. Computer therapy for the anxiety and depressive disorders is effective, acceptable, and practical health care: A meta-analysis. PLoS ONE. 2010;5. 
13. El-Mahalli AA, El-Khafif SH, Al-Qahtani MF. Successes and challenges in the implementation and application of telemedicine in the eastern province of Saudi Arabia. Perspect Health Inf Manag. 2012;9:1-27.

14. Dick PT, Filler R, Pavan A. Participant satisfaction and comfort with multidisciplinary pediatric telemedicine consultations. J Pediatr Surg. 1999;34:137-42.

15. Shore JH, Brooks E, Savin DM, Manson SM, Libby AM. An economic evaluation of telehealth data collection with rural populations. Psychiatr Serv. 2007;58:830-5.

16. Kothapalli P, Bove AA, Santamore WP, Homko C, Kashem $A$. Factors affecting frequency of patient use of internet-based telemedicine to manage cardiovascular disease risk. J Telemed Telecare. 2013;19:205-8.

17. Polisena J, Tran K, Cimon K, Hutton B, McGill $\mathrm{S}$, Palmer $\mathrm{K}$, et al. Home telemonitoring for congestive heart failure: A systematic review and meta-analysis. J Telemed Telecare. 2010;16:6876.

18. Hassan E. Tele-ICU and patient safety considerations. Crit Care Nurs Q. 2018;41:4759.

19. Shahbaz R, Salducci M. Law and order of modern ophthalmology: Teleophthalmology, smartphones legal and ethics. Eur J Ophthalmol. 2021;31:13-21.

20. Whited JD, Hall RP, Foy ME, Marbrey LE, Grambow SC, Dudley TK, et al. Patient and clinician satisfaction with a store-and-forward teledermatology consult system. Telemed J e-Health. 2004;10:422-31.

21. Baruffaldi F, Gualdrini G, Toni A. Comparison of asynchronous and realtime teleconsulting for orthopaedic second opinions. J Telemed Telecare. 2002;8:297-301.

22. Canberk S, Behzatoglu K, Caliskan CK, Gelmez S, Kayhan KC, Aydemir SF, et al. The role of telecytology in the primary diagnosis of thyroid fine-needle aspiration specimens. Acta Cytol. 2020;64:323-31.

23. Lu K, Marino NE, Russell $D$, Singareddy A, Zhang $D$, Hardi A, et al. Use of short message service and smartphone applications in the management of surgical patients: A systematic review. Telemed e-Health. 2018;24:406-14.

24. Funderskov KF, Boe Danbjørg D, Jess M, Munk $L$, Olsen Zwisler AD, Dieperink KB. Telemedicine in specialised palliative care: Healthcare professionals' and their perspectives on video consultations: A qualitative study. J Clin Nurs. 2019;28:3966-76.

25. Maarop N. Teleconsultation technology and its benefits: In the case of public hospitals in Malaysia. CONF-IRM 2011 Proc. 2011;1-12.

26. Gajarawala SN, Pelkowski JN. Telehealth benefits and barriers. J Nurse Pract. 2020;

27. World Health Organization. Coronavirus [Internet]. World Health Organization. 2020. Available from: https://www.who.int/healthtopics/coronavirus\#tab=tab_3

28. Huang $C$, Wang $Y$, Li $X$, Ren L, Zhao J, Hu Y, et al. Clinical features of patients infected with 2019 novel coronavirus in Wuhan, China. Lancet. 2020;395:497-506.

29. Smith AC, Thomas E, Snoswell CL, Haydon $H$, Mehrotra A, Clemensen J, et al. Telehealth for global emergencies: Implications for Coronavirus Disease 2019 (COVID-19). J Telemed Telecare. 2020;26:309-13.

30. Wax RS, Christian MD. Practical recommendations for critical care and anesthesiology teams caring for novel coronavirus (2019-nCoV) patients. Can J Anesth [Internet]. 2020;67:568-76. Available from: https://doi.org/10.1007/s12630-02001591-x

31. Zhou $X$, Snoswell $C L$, Harding $L E$, Bambling $M$, Edirippulige $S$, Bai $X$, et al. The role of telehealth in reducing the mental health burden from COVID-19. Telemed e-Health. 2020;26:377-9.

32. Octavius GS, Antonio F. Antecedents of intention to adopt mobile health (mHealth) application and its impact on intention to recommend: An evidence from Indonesian customers. Int. J. Telemed. Appl. 2021; Article ID 6698627:124. 